\title{
Aktuelle Entwicklungen an den österreichischen
}

\section{Bibliotheken 2016}

Zusammenfassung: Im vorliegenden Beitrag wird über aktuelle Projekte und Trends im österreichischen Bibliothekswesen im Jahr 2016 berichtet. Vorgestellt werden Verbundprojekte und Kooperationsprojekte österreichischer Bibliotheken, aktuelle Projekte der Österreichischen Nationalbibliothek und des Forums Universitätsbibliotheken Österreichs. Angesprochen werden auch Themen wie Forschungsdaten, Open Access, NS-Provenienzforschung, Bibliotheksausbildung, Tagungen und Publikationen der Vereinigung Österreichischer Bibliothekarinnen und Bibliothekare sowie Bibliotheks- und Informationspolitik in Österreich.

Schlüsselwörter: Österreich; Bibliothekswesen; Entwicklung; 2016

\section{Current Developments in Austrian Libraries in 2016}

Abstract: The present contribution relates about current projects and trends in Austrian librarianship in 2016. Network projects and cooperation projects are presented as well as important projects of the Austrian National Library and of the Council of Austrian University Libraries. Themes such as Open Access, research data, provenance research of Nazilooted property, library education, conferences and publications of the Austrian Association of Librarians and library and information policy in Austria are mentioned.

Keywords: Austria; librarianship; development; 2016

\section{1 Österreichischer Bibliothekenverbund}

\subsection{Neues Bibliothekssystem Alma}

Nach einem zweijährigen Vergabeverfahren für ein neues Bibliotheksverbundsystem mit lokalen, zentralen und konsortialen Funktionalitäten fiel im September 2015 die Entscheidung für das System Alma der Firma Ex Libris. ${ }^{1}$ Im Januar 2016 wurde mit der Implementierung des neuen Systems an der Österreichischen Bibliothekenverbund und Service Gesellschaft m.b.H. (OBVSG) und den 13 am Verfahren beteiligten Bibliotheken begonnen. Im ersten Halbjahr wurde erfolgreich eine Konzeptionsphase umgesetzt, in der die Spezifikation zwingender Konzeptanforderungen festgelegt

\footnotetext{
${ }^{1}$ Hamedinger, Wolfgang (2016): Austrian Library Network and Next Generation Library System: Alma. In: BibliothekForschung und Praxis, 40 (3), 341-346.
} 
wurde. In 32 Konzeptgruppen mit jeweils einem Vertreter der OBVSG, der Anbieterfirma sowie eigenen Expertengruppen wurden essentielle Themen, wie Verbundarchitektur, Normdaten, ZDB, Datenschutz, Migration \& Parallelbetrieb sowie konsortiale Funktionalitäten, bearbeitet. Weiters wurde eine Arbeitsgruppe beauftragt, ein Schulungskonzept zu entwickeln. ${ }^{2}$ Im August 2016 begann die Implementierungsphase, in der vorbereitende Aktivitäten gesetzt wurden, die Themen wie Testumgebung, Training, Studium der Dokumentation, Überprüfung von Arbeitsabläufen und Daten gewidmet waren. Im November 2016 begann die eigentliche Implementierungsphase, die in drei Schüben realisiert wird. Der Umstieg von Gruppe 1, bestehend aus sechs Bibliotheken, auf das neue Bibliothekssystem Alma wird im August 2017 erfolgen, Gruppe 2, bestehend aus sieben Bibliotheken, wird im Januar 2018 umsteigen; zeitgleich werden auch die Arbeiten für Gruppe 3, in der zentrale Services der OBVSG implementiert werden, beginnen. ${ }^{3}$

\subsection{Weitere Aktivitäten der OBVSG}

Für die Gemeinsame Normdatei (GND), die an der Deutschen Nationalbibliothek vorgehalten und von allen deutschsprachigen Bibliotheksverbünden sowie weiteren Institutionen kooperativ betrieben wird, wurde an der OBVSG eine GND-Koordinationsstelle eingerichtet, die für die nationale Zusammenarbeit zuständig ist. ${ }^{4} 5$

Das kooperative Modell für die Bearbeitung der Datensätze in der Zeitschriftendatenbank (ZDB) im Österreichischen Bibliothekenverbund wurde 2016 laufend erweitert. Das webbasierte System WebCat ermöglicht es Einrichtungen mit kleineren Zeitschriftenbeständen, die eigenen Titeldaten über einen browserbasierten Zugang mittels Formularen in der ZDB zu erfassen. ${ }^{67}$

In der Verbundsuchmaschine des Österreichischen Bibliothekenverbundes wurde ein zentraler Nachweis für alle in Österreich erscheinenden Hochschulschriften etabliert. ${ }^{8} 9$

\footnotetext{
2 OBV (2016): Ein langer Weg beginnt mit dem ersten Schritt: Vier Monate Alma-Implementierung im OBV. In: OBV SG Newsletter. Aktuelles aus dem Österreichischen Bibliothekenverbund (OBV), (1), .2.

${ }^{3}$ OBV (2016): Gute Vorbereitung ist der halbe Weg: Zwölf Monate Alma-Implementierung im OBV. In: OBV SG Newsletter. Aktuelles aus dem Österreichischen Bibliothekenverbund (OBV), (2), 2.

${ }^{4}$ OBV (2016): Normdaten und ihr enormer Nutzen: Die GND-Koordinationsstelle an der OBVSG. In: OBV SG Newsletter. Aktuelles aus dem Österreichischen Bibliothekenverbund (OBV), (2), 3.

${ }^{5}$ Klien, Peter (2016): Mitteilungen aus der OBVSG. In: Mitteilungen der Vereinigung Österreichischer Bibliothekarinnen \& Bibliothekare, 69 ((3), 487-491.

${ }^{6}$ OBV (2016): Kollektive Weisheit am Vormarsch: Steter Zuwachs für das kooperative ZDB-Modell im OBV. In: OBV SG Newsletter. Aktuelles aus dem Österreichischen Bibliothekenverbund (OBV), (1), 3.

7 OBV (2016): Kooperatives ZDB-Modell wächst. In: OBV SG Newsletter. Aktuelles aus dem Österreichischen Bibliothekenverbund (OBV), (2), 2.

8 OBV (2016): Alles an einem Ort: Hochschulschriften in der Verbundsuchmaschine. In: OBV SG Newsletter. Aktuelles aus dem Österreichischen Bibliothekenverbund (OBV), (2), 1.

${ }^{9}$ Klien, Peter (2016): Mitteilungen aus der OBVSG. In: Mitteilungen der Vereinigung Österreichischer Bibliothekarinnen \& Bibliothekare, 69 (1) 100-102.
} 
2016 ist die Zahl der Anwenderbibliotheken von Visual Library um fünf weitere Institutionen gewachsen. ${ }^{10}$ Unter der Bezeichnung BOKU:EPUB wurde an der Universität für Bodenkultur eine Visual Library-Anwendung installiert, die als Open-Access-Plattform für elektronische Publikationen und digitalisierte Medien an der Universität fungiert und von Universitätsbibliothek und Universitätsarchiv betrieben wird. ${ }^{11}$

Der 12. Verbundtag, der am 12. Mai 2016 an der Wirtschaftsuniversität Wien stattgefunden hat und an dem zirka 140 Personen teilgenommen haben, war vor allem dem neuen

Bibliotheksverbundsystem Alma gewidmet. ${ }^{12} 13$

\section{Kooperation E-Medien Österreich}

Die Kooperation E-Medien Österreich (KEMÖ) agiert auf der Basis des von insgesamt 55 Konsortialpartnern abgeschlossenen Kooperationsvertrages (Laufzeit: 2015-2017). Die Liste der Mitglieder umfasst österreichische Universitäten und Privatuniversitäten, Fachhochschulen, Landesbibliotheken, die Österreichische Nationalbibliothek sowie sonstige öffentliche und private Informations-, Forschungs-und Bildungseinrichtungen mit Sitz in Österreich. Die Geschäftsstelle der KEMÖ, an der drei Personen beschäftigt sind, ist als Abteilung der OBVSG zugeordnet. Aktuell werden etwa 70 Produktverträge für elektronische Medien für die Konsortialpartner administriert, wobei in den letzten Jahren auch das Thema Open Access immer stärker in den Fokus der Verhandlungen und Verträge mit den Verlagen gerückt ist (siehe Abschnitt 6).

\section{3 Österreichische Nationalbibliothek}

\subsection{Titel in ANNO online}

Zum Zeitpunkt der Freischaltung des digitalen Zeitschriftenlesesaals der Österreichischen Nationalbibliothek 2003 umfasste das Angebot des neuen Digitalisierungsprojektes ANNO 15 Zeitschriften. Im Dezember 2016 wurde der tausendste Zeitschriftentitel digitalisiert und online verfügbar gemacht. ANNO umfasst nunmehr mehr als 17 Millionen Zeitungs- und Zeitschriftenblätter vom 16. bis zum 20. Jahrhundert in zwanzig unterschiedliche Sprachen. Die meistgelesene Zeitschrift ist die Wiener Zeitung, deren Vorläuferin unter dem Titel Wienerisches Diarium 1703 erstmals

\footnotetext{
${ }^{10}$ OBV (2016): Ständig neue Kunden und Funktionen: Retrodigitalisierung und Publikationsserver mit Visual Library. In: OBV SG Newsletter. Aktuelles aus dem Österreichischen Bibliothekenverbund (OBV), (2), 3.

${ }^{11}$ OBV (2016): Was ist BOKU:EPUB? Der neue Publikationsserver der Universität für Bodenkultur. In: OBV SG Newsletter. Aktuelles aus dem Österreichischen Bibliothekenverbund (OBV), (2), 3.

12 OBV (2016): Verbundtag 2016 an der Wirtschaftsuniversität (WU) in Wien. In: OBV SG Newsletter. Aktuelles aus dem Österreichischen Bibliothekenverbund (OBV), (1), 1.

${ }^{13}$ Klien, Peter (2016): Mitteilungen der OBVSG. In: Mitteilungen der Vereinigung Österreichischer Bibliothekarinnen \& Bibliothekare, 69 (2), 314-318.
} 
erschienen ist. ${ }^{14}$ ANNO wird laufend hinsichtlich Umfang und Funktionalitäten - Stichwort Volltextsuche - erweitert, was sich auch in einer sehr starken Nutzung dieses digitalen Angebotes zeigt. $^{15}$

\subsection{Digitaler Prunksaal und Bestände aus dem Prunksaal online}

Seit Juli 2016 kann der Prunksaal der Österreichischen Nationalbibliothek, die zwischen 1723 und 1726 nach den Plänen des Hofarchitekten Johann Bernhard Fischer von Erlach (1656-1723) errichtet worden ist, online, kostenlos und rund um die Uhr von allen interessierten Personen besucht werden. ${ }^{16}$

Seit Dezember 2016 sind auch die 200.000 Bücher des Prunksaals der Österreichischen Nationalbibliothek - diese sind zwischen 1501 und 1850 erschienen - online verfügbar. Dieser Bestand ist Teil des historischen Druckschriftenbestands der Österreichischen Nationalbibliothek, der rund 600.000 Werke umfasst und zu den fünf bedeutendsten Sammlungen historischer Bücher weltweit zählt. Die Digitalisierung dieses Bestandes erfolgt seit 2010 im Rahmen des Projektes „Austrian Books Online“ $(A B O)$ in einer Public-Private-Partnership der Nationalbibliothek mit Google. Mittlerweile wurden bereits mehr als 420.000 Bände gescannt. Die Digitalisate sind über den Onlinekatalog der Österreichischen Nationalbibliothek, über Europeana und über Google Books recherchierbar, die Volltexte können kostenfrei durchsucht, gelesen und auch vollständig heruntergeladen werden.Der Abschluss des Projektes $A B O$, in dessen Rahmen insgesamt rund 200 Millionen Seiten digitalisiert werden, ist für 2018 geplant. ${ }^{17}$

\subsection{Crowdfunding-Aktion für Prachtband zu Maria Theresia}

Nach dem Aufruf zur Beteiligung an der Restaurierung und Digitalisierung eines Prachtbandes zu Maria Theresia (1717-1780) am 20. Oktober 2016 konnte schon nach 24 Tagen das Finanzierungsziel von 15.500 Euro deutlich übertroffen werden. Die Nationalbibliothek nutzte für ihre erste Crowdfunding-Aktion die Plattform wemakeit.com, allerdings erfolgten auch zahlreiche Spenden über Erlagschein und Direktüberweisungen. ${ }^{18}$

\footnotetext{
${ }^{14}$ Pauser, Josef (2016): ÖNB: 1000 Titel in ANNO online! In: VÖBBLOG, 22.12.2016. Online: http://www.univie.ac.at/voeb/blog/?p=42409

${ }^{15}$ Müller, Christa (2016): ANNO - Der digitale Zeitungslesesaal der Österreichischen Nationalbibliothek. In: Bibliothek Forschung und Praxis, 40 (1), 83-89.

16 Pauser, Josef (2016): ÖNB: Den Prunksaal der Österreichischen Nationalbibliothek online besuchen. In: VÖBBLOG, 20.07.2016. Online: http://www.univie.ac.at/voeb/blog/?p=40955

17 Die Presse (2016): Die Bestände aus dem Prunksaal der ÖNB sind online. 200.000 wertvolle Bände sind digitalisiert und ab sofort abrufbar. In: Die Presse, 31.12.2016. Online: http://diepresse.com/home/kultur/literatur/5143718/Die-Bestaendeaus-dem-Prunksaal-der-OeNB-sind-online

18 Pauser, Josef (2016): ÖNB: Crowdfunding-Aktion der Österreichischen Nationalbibliothek erfolgreich abgeschlossen. In: VÖBBLOG, 22.11.2016. Online: http://www.univie.ac.at/voeb/blog/?p=42105
} 


\subsection{Webarchivierung}

Nachdem das Web@archiv Österreich bereits 2008 begründet worden ist, erfolgte mit dem 2009 in Kraft getretenen österreichischen Mediengesetz eine Ermächtigung der Österreichischen Nationalbibliothek zur Sammlung und Präsentation von Online-Inhalten. Bis 2016 wurde ein Datenvolumen von ca. 75 Terabyte gesammelt. Die Anzahl der Dateien betrug nahezu 2,5 Milliarden; diese sind 1,67 Millionen unterschiedlichen Internetadressen zuzuordnen. Der Sammelauftrag bezieht sich auf Dokumente im Word Wide Web mit Österreichbezug, was sich in der praktischen Durchführung als schwierig erweist. Eine Gleichsetzung des österreichischen Kulturgutes mit der nationalen .at-Domäne ist nicht zielführend. ${ }^{19}$

\subsection{Buchpatenschaften}

Anlässlich des 25jährigen Jubiläums der Aktion Buchpatenschaft konnte die Österreichische Nationalbibliothek mit dem Star-Tenor Jonas Kaufmann einen prominenten Buchpaten gewinnen. ${ }^{20}$

\subsection{Forschungstätigkeit und Forschungsblog der ÖNB}

Die Österreichische Nationalbibliothek sieht ihre Aufgabe als große Gedächtnis-Institution zunehmend - neben den bisherigen quellenorientierten oder hypothesengetriebenen Forschungen in Aktivitäten der Digital Humanities, die auch zur Demokratisierung des Wissens beitragen. Quellen werden mittels digitaler Medien verbreitete und erschlossen und auch die Forschungsergebnisse werden im Internet präsentiert. ${ }^{21}$

In diesem Kontext ist auch der Forschungsblog der Österreichische Nationalbibliothek zu sehen, der im November 2016 mit der Zielsetzung gestartet wurde, Einblick in die aktuellen

Forschungsvorhaben etwa im Bereich der Bestandserschließung oder von Restaurierungsvorhaben zu geben und Hintergrundinformationen zu Ausstellungen und Veranstaltungen bieten. ${ }^{22}$

\subsection{ORF III-Dokumentation über Österreichische Nationalbibliothek}

ORF III brachte am 18. Oktober 2016 in der Reihe Mythen der Geschichte eine Dokumentation unter dem Titel Lebensraum Nationalbibliothek, in der die Geschichte der Bibliothek von Anfängen im Jahr 1368, als mit dem Evangeliar des Johannes von Troppau (das erste Buch an die Bibliothek gelangte, bis in die Gegenwart. ${ }^{23}$

\footnotetext{
${ }^{19}$ Mayr, Michaela; Predikaka, Andreas: Nationale Grenzen im World Wide Web - Erfahrungen bei der Webarchivierung in der Österreichischen Nationalbibliothek. In: Bibliothek - Forschung und Praxis, 40 (1), 90-95.

${ }^{20}$ Pauser, Josef (2016): Buchpatenschaft durch Star-Tenor Jonas Kaufmann. In: VÖBBLOG, 10.04.2016. Online: http://www.univie.ac.at/voeb/blog/?p=40025

${ }^{21}$ Fetz, Bernhard (2016): Stilus, Bleistift und Computer - Zur Forschungstätigkeit an der Österreichischen Nationalbibliothek. In: Bibliothek - Forschung und Praxis, 40 (1), 96-103.

22 Österreichische Nationalbibliothek (2016): Forschungsblog. Online: https://www.onb.ac.at/de/forschung/forschungsblog/

${ }^{23}$ Lebensraum Nationalbibliothek (2016). In: Mythen der Geschichte, Folge 125. Online: https://www.fernsehserien.de/mythen-der-geschichte-a/folgen/125-lebensraum-nationalbibliothek-996047
} 


\subsection{Ausstellungen}

2016 wurden im Prunksaal der Österreichischen Nationalbibliothek drei Ausstellungen präsentiert. ${ }^{24}$ Von November 2015 bis Februar 2016 wurden 80 einzigartige Exponate aus über 200 Jahren Buchkultur im Rahmen von Goldene Zeiten: Meisterwerke der Buchkunst von der Gotik bis zur Renaissance gezeigt, darunter die Goldene Bulle.

In der Ausstellung Der ewige Kaiser. Franz Joseph I. 1830-1916 wurden von März bis November 2016 zahlreiche Exponate über Kaiser Franz Joseph (1830-1916) präsentiert. Neben Kinderzeichnungen und Schreibübungen des späteren Kaisers und Briefen an seine langjährige Freundin Katharina Schratt (1853-1940) wurde etwa eine zehn Meter lange Installation mit 86 Porträts aus 86 Lebensjahren gezeigt, die zu einer Zeitreise in die Habsburger Monarchie einlud. Die Eröffnung der Ausstellung erfolgte am 10. März 2016 durch Bundespräsident Heinz Fischer, Bundesminister Josef Ostermayer und Generaldirektorin Johanna Rachinger. ${ }^{25}$

Die abschließende Ausstellung 2016 im Prunksaal war dem Thema Unter Bethlehems Stern gewidmet. Von Dezember 2016 bis Januar 2017 wurden 24 Prachthandschriften des Mittelalters gezeigt. $^{26}$

Mit der Sonderausstellung Hieroglyphen und Alphabete, in der Schriftstücke von Schülern und Lehrern aus über 2.500 Jahren Unterricht präsentiert wurden und die von Juni 2016 bis Januar 2017 im Papyrusmuseum gezeigt wurde, hatte die Nationalbibliothek ein weiteres Highlight in ihrem Ausstellungsprogramm. ${ }^{27}$

\subsection{Staatsgäste im Prunksaal}

2016 wurde an der Österreichischen Nationalbibliothek anlässlich der Übernahme des Vorsitzes in der Organisation für Sicherheit und Zusammenarbeit in Europa (OSZE) durch Deutschland ein Empfang ausgerichtet, in dessen Rahmen von Generaldirektorin Johanna Rachinger eine Buchpatenschaft für einen Stich mit der Stadtansicht von Hamburg an den deutschen Außenminister und OSZE-Chairperson-in-Office Frank-Walter Steinmeier übergeben wurde.

\footnotetext{
24 Österreichische Nationalbibliothek Prunksaal (2016): Vergangene Ausstellungen. Online: https://www.onb.ac.at/museen/prunksaal/sonderausstellungen/vergangene-ausstellungen/

${ }^{25}$ Pauser, Josef (2016): Eröffnung der Ausstellung „Der ewige Kaiser. Franz Joseph I. 1830-1916“. In: VÖBBLOG, 12.03.2016. Online: http://www.univie.ac.at/voeb/blog/?p=39759

${ }^{26}$ Pauser, Josef (2016): ÖNB-Ausstellung: Unter Bethlehems Stern (7.12.2016-15.1.2017). In: VÖBBLOG, 28.11.2016. Online: http://www.univie.ac.at/voeb/blog/?p=42138

27 Pauser, Josef (2016): ÖNB: Hieroglyphen und Alphabete - 2.500 Jahre Unterricht im alten Ägypten (16. Juni $2016-8$. Jänner 2017). In: VÖBBLOG, 16.06.2016. Online: http://www.univie.ac.at/voeb/blog/?p=40663
} 
Besucht wurde die Nationalbibliothek auch von S.E. Maithripala Sirisena, dem Präsidenten der Republik Sri Lanka, besucht. ${ }^{z 8}$

\subsection{Forum Universitätsbibliotheken Österreichs}

Das Forum Universitätsbibliotheken Österreichs (ubifo), ein Kooperationsgremium der Leiterinnen und Leiter der 20 Universitätsbibliotheken der öffentlichen Universitäten sowie der Österreichischen Nationalbibliothek, veranstaltete 2016 zwei Meetings (09.03.2016 an der Universität Wien, 16.11.2016 an der Universität für Musik und darstellende Kunst Wien). Folgende Themen standen dabei im Fokus: die Implementierung des neuen Bibliothekssystems Alma (siehe Abschnitt 1), das Hochschulraumstrukturmittel-Projekt E-Infrastructures Austria (2014-2016), die Hochschulraumstrukturmittel-Projekteinreichungen Austrian Transition to Open Access (AT2OA) (siehe Abschnitt 6), und e-Infrastructures Austria Plus (siehe Abschnitt 5), die zukünftige Ausrichtung der Kooperation E-Medien Österreich im Hinblick auf den Folgevertrag 2018-2020, Linked Open Data, Zentralredaktion Formalerschließung, Kooperationsmöglichkeiten hinsichtlich der Aufbereitung und Zurverfügungstellung von barrierefreier Literatur, Ablieferung elektronischer Dissertationen, Alternativen für zukünftige Leistungsmessung an Bibliotheken nach dem Ende des Bibliothkesindex $(B I X)$ sowie NS-Provenienzforschung.

Am 27. und 28. April 2016 wurde im ParkschlössI Wien eine Klausur zum Thema ubifo Strategie 2016-2018 abgehalten.

\section{E-Infrastructures Austria}

An E-Infrastructures Austria, ${ }^{29}$ einem vom Bundesministerium für Wissenschaft, Forschung und Wirtschaft (BMWFW) finanzierten Hochschulraumstrukturmittelprojekt (Laufzeit 2014-2016), beteiligen sich 25 Projektpartner, ein assoziierter Projektpartner und fünf Institutionen mit Beobachterstatus.

2016 wurden zwei zweitägige Generalversammlungen des Projektmanagements mit den Projektpartnern (14.04.2016 bzw. 18.11.2016, jeweils an der Universität Wien) durchgeführt.

Im letzten Jahr konnten die drei Teilprojekte des HRSM-Projektes e-Infrastructures Austria abgeschlossen werden. An den 25 Partnereinrichtungen wurden als In-Kind-Leistungen Repositorien aufgebaut bzw. befanden sich in Planung (Teilprojekt A). 2016 wurde von einer ExperInnengruppe eine Muster-Policy für Forschungsdatenmanagement erarbeitet; an einzelnen der Partnereinrichtungen wurden Forschungsdatenmanagementpläne erprobt (Teilprojekt B). Das Ziel,

\footnotetext{
28 ÖNB Magahzin (2016): Hoher Besuch. Internationale Gäste gaben sich in der Österreichischen Nationalbibliothek. In: Österreichische Nationalbibliothek Magazin (1/April 2016), 21.

${ }^{29}$ E-Infrastructures Austria (2016). Online: http://www.e-infrastructures.at
} 
Fortbildung und Vernetzung für Forschungsdatenmanagement zu ermöglichen, wurde im Berichtsjahr in Form von Workshops zu den Themen Metadaten und Langzeitarchivierung und insbesondere durch ein viertätiges Seminar für den professionellen Umgang mit Forschungsdaten erfolgreich umgesetzt (Teilprojekt C). ${ }^{30} 31$

Neben dem Fortbildungsseminar, das von 6. bis 9. Juni 2016 an der Universität Wien stattgefunden hat ${ }^{32}$, wurde am 6. April 2016 auch ein internationaler Workshop zum Thema ForschungsdatenPolicies im Rahmen des EU-Projektes LEARN an der Universität Wien organisiert. ${ }^{33}$ Weitere Ergebnisse des dritten Projektjahres von e-Infrastructures Austria waren Workshops zu den Themen Langzeitarchivierung ${ }^{34}$ und Metadaten sowie die Erstellung einer Guideline zur Langzeitarchivierung ${ }^{35}$ sowie eine Expertise zu den Auswirkungen des neuen Urheberrechts auf den Betrieb und die Nutzung von Repositorien. ${ }^{36}$

Zur Dokumentation der Aktivitäten und Ergebnisse von e-Infrastructures Austria wurde im letzten Projektjahr ein umfassender Bericht vorgelegt. ${ }^{37}$

Zum Zeitpunkt des Projektstarts war in der österreichischen Hochschul- und Forschungslandschaft nur wenig Wissen zu den Themen Repositorien und Forschungsdaten vorhanden. Im Rahmen von eInfrastructures Austria wurden diese zukunftsweisenden Themen erfolgreich aufgegriffen und nachhaltig etabliert. Den überzeugenden Beweis für diese Einschätzung liefert der Use Case Austria im Rahmen der European Open Science Cloud, in dem sich ab 2017 insgesamt zehn Projekte laufend

\footnotetext{
${ }^{30}$ Bauer, Bruno; Sánchez Solís, Barbara (2016): e-Infrastructures Austria 2014-2016: Ein österreichweites Projekt für den koordinierten Aufbau und die Weiterentwicklung von Repositorieninfrastrukturen. In: Bruno Bauer, Andreas Ferus und Josef Pauser (Hrsg.): Offen(siv)e Bibliotheken: Neue Zugänge, neue Strukturen, neue Chancen. 32. Österreichischer Bibliothekartag, Wien, 15.-18. September 2015. Wolfgang Neugebauer Verlag GesmbH: Graz/Feldkirch, 2016, S. 25-40. (Schriften der Vereinigung Österreichischer Bibliothekarinnen und Bibliothekare, 14)

${ }^{31}$ Bauer, Bruno; Budroni, Paolo; Ferus, Andreas; Ganguly, Raman; Ramminger, Eva; Sánchez Solís, Barbara (2016): eInfrastructures Austria 2015: Bericht über das zweite Jahr des Hochschulraumstrukturmittelprojekts für den koordinierten Aufbau und die kooperative Weiterentwicklung von Repositorieninfrastrukturen. In: Mitteilungen der Vereinigung Österreichischer Bibliothekarinnen \& Bibliothekare, 69 (3/4), 9-40.

32 Budroni, Paolo; Flicker, Katharina; Sánchez Solís, Barbara (2016): e-Infrastructures Austria - Fortbildungsseminar für Forschungsdaten und e-Infrastrukturen (Wien, 6.-9. Juni 2016). In: Mitteilungen der Vereinigung Österreichischer Bibliothekarinnen \& Bibliothekare, 69 (3/4), 492-500.

${ }^{33}$ Traub, Imola Dora; Sánchez Solis, Barbara; Budroni, Paolo (2016): Forschungsdaten und zeitgemäße Aufarbeitung durch Policies - 2. internationaler LEARN Workshop zum Thema 'Forschungsdatenmanagement' (Wien, 6. April 2016). In: Mitteilungen der Vereinigung Österreichischer Bibliothekarinnen \& Bibliothekare, 69 (1), 142-150.

34 Mayer, Adelheid (2016): Workshop „Software-Lösungen zur Langzeitarchivierung und Repositorien-Verwaltung aus Anwendersicht" (Wien, 21. April 2016) . In: Mitteilungen der Vereinigung Österreichischer Bibliothekarinnen \& Bibliothekare, 69 (3/4), 151-154.

35 Birkner, Michael; Gonter, Gerhard; Lackner, Karin; Kann, Bettina; Kranewitter, Michael; Mayer, Adelheid; Parschalk, Andreas (2016): Guideline zur Langzeitarchivierung. In: Mitteilungen der Vereinigung Österreichischer Bibliothekarinnen \& Bibliothekare, 69 (3/4), 41-57.

${ }^{36}$ Amini, Seyavash; Hamdi, Djawaneh; Huß, Andreas (2016): Auswirkungen des „neuen Urheberrechts“ auf den Betrieb und die Nutzung elektronischer Repositorien. In: Mitteilungen der Vereinigung Österreichischer Bibliothekarinnen \& Bibliothekare, 69 (3/4), 397-413.

${ }^{37}$ E-Infrastructures Austria (Hrsg., 2016): Projektbericht 2014-2016, Version 2.0. Wien, Jänner 2017. Online:

https://phaidra.univie.ac.at/detail_object/o:460779
} 
zum Themenkomplex Open Science austauschen werden. Als unmittelbare Fortsetzung von $e$ Infrastructures Austria wurde vom BMWFW das Folgeprojekt e-Infrastructures Austria Plus bewilligt, in dessen Rahmen Maßnahmen im Kontext von Forschungsdatenmanagement an neun Universitäten gefördert werden.

E-Infrastructures Austria hat in den drei Projektjahren beeindruckende Dimensionen erreicht, wozu auch eine gut abgestimmte Governance der Projektpartnereinrichtungen eine wesentliche Voraussetzung gebildet hat. ${ }^{38}$ Am Netzwerk waren mehr als 100 Personen beteiligt, 23 Workshops und Veranstaltungen wurden organisiert, 27 Vorträge und Präsentationen im Rahmen von nationalen und internationalen Tagungen und Kongressen gehalten sowie 15 Beiträge in nationalen und internationalen Fachzeitschriften publiziert. Rechnet man den zeitlichen Aufwand der Arbeitsgruppen die Generalversammlungen, der Workshops und Schulungen zusammen, so wurde von den Mitarbeiterinnen und Mitarbeitern der Projektpartnereinrichtungen insgesamt 14.644 Stunden bzw. 1.830 Arbeitstage eingebracht, wobei die Arbeitsleistung des Projektmanagements, der Reiseaufwand sowie die lokalen Personalressourcen für Teilprojekt A hier nicht miteingerechnet sind.

\section{Open Access}

\subsection{Open Access Network Austria}

Dem 2012 initiierten Open Access Network Austria (OANA) gehören Wissenschaftlerinnen und Wissenschaftler sowie Vertreterinnen und Vertretern von Forschungsförderinstitutionen, Rektoraten und Universitätsbibliotheken sowie des für Wissenschaft und Forschung zuständigen Bundesministeriums, an.

Am 1. Juni 2016 fand die mittlerweile bereits 3. OANA-Informationsveranstaltung im Palais Harrach in Wien statt, in dessen Rahmen aktuelle Entwicklungen im Kontext von Open Access in Österreich vorgestellt wurden. Weitere Präsentationen waren den Ergebnissen der OANA Arbeitsgruppen im vergangenen Jahr gewidmet, die sich mit den Themen Finanzierung von Open Access, Nationale Open Access Strategie, Open Access und Urheberrecht sowie Open Access und Scholarly Communication auseinandergesetzt haben. ${ }^{39}$

Von der OANA AG Open Science wurden 2016 The Vienna Principles: A Vision for Scholarly Communication in the 21st Century veröffentlicht. ${ }^{40} 41$

\footnotetext{
${ }^{38}$ Budroni, Paolo (2016): Good Governance. Strukturen zur Sicherung der Nachhaltigkeit von Projekten im akademischen Umfeld. Das nationale Projekt e-Infrastructures Austria. In: ABI Technik, 36 (1), 24-32.

${ }^{39}$ Rieck, Katharina; Matt, Ina (2016): 3. Informationsveranstaltung des Open Access Network Austria (OANA) (Wien, 1. Juni 2016) . In: Mitteilungen der Vereinigung Österreichischer Bibliothekarinnen \& Bibliothekare, 69 (2), 337-345.

${ }^{40}$ Kraker, Peter; Dörler, Daniel; Ferus, Andreas; Gutounig, Robert; Heigl, Florian; Kaier, Christian; Rieck, Katharina; Šimukovič, Eöema: Vignoli, Michela (2016): The Vienna Principles: A Vision for Scholarly Communication in the 21st Century. 2016. Online: http://viennaprinciples.org/v1/
} 


\subsection{Open Access Policies}

Die Berliner Erklärung wurde bis Ende 2016 von elf von 21 öffentlichen Universitäten sowie von sechs weiteren österreichischen Privatuniversitäten und Fachhochschulen unterzeichnet. Zu den Unterzeichnerinnen der Berliner Erklärung zählen auch die Universitätenkonferenz (2004), der FWF (2003), das Institute of Science and Technology Austria (2014), der Österreichische Wissenschaftsrat (2015), der OeAD GmbH - Österreichischer Austauschdienst (2016), die acib GmbH - Austrian Centre of Industrial Biotechnology (2016) und das AIT - Austrian Institute of Technology GmbH (2016).

2016 hat sich auch die Zahl der öffentlichen Universitäten, die eine Open Access Policy veröffentlicht haben, von fünf auf neun Einrichtungen erhöht (Universität Graz, Universität Klagenfurt, Universität Salzburg, Universität Wien, Technische Universität Graz, Universität für Bodenkultur Wien, Veterinärmedizinische Universität Wien, Wirtschaftsuniversität Wien, Akademie der bildenden Künste Wien). Die Policies der genannten Einrichtungen wurden analysiert und daraus eine Checkliste mit Textbausteinen erstellt, die für die Entwicklung zukünftiger Open Access Policies weiterer Forschungsinstitutionen als Orientierungshilfe zur Verfügung steht. Die untersuchten Policies enthalten Unterstützungserklärungen für die Berliner Erklärung sowie Empfehlungen oder Ermutigungen für die Gold Road to Open Access. Häufig in den Policies angesprochen werden die Themen Publikationsfonds, hybrides Open Access und Green Road to Open Access (Repositorien). In einigen Policies finden sich auch Anmerkungen zu den Themen Publikations- und Wissenschaftsfreiheit, zu Forschungsdaten, zu monografischen Veröffentlichungen und zu Urheberrechten. Gelegentlich thematisiert werden auch die Mitarbeit an Open Access-Zeitschriften und die Unterstützung für das Open Access-Publikationsmodell, die Information und Beratung der Wissenschaftlerinnen und Wissenschaftler durch die Institution, Anreize für Wissenschaftlerinnen und Wissenschaftler, die Digitalisierung des digitalen kulturellen Erbes sowie die Beteiligung an Open Access-Kooperations- und Koordinationsaktivitäten. ${ }^{42}$

\subsection{Open Access Vereinbarungen mit Modellcharakter}

Unter den seit 2014 über die Kooperation E-Medien Österreich (KEMÖ) abgeschlossenen modellhaften Konsortialvereinbarungen mit Verlagen für den Zugriff auf elektronische Zeitschriften für österreichische Hochschul- und Forschungseinrichtungen und die Möglichkeit zur Open Access-

\footnotetext{
${ }^{41}$ Kraker, Peter; Dörler, Daniel; Ferus, Andreas; Gutounig, Robert; Heigl, Florian; Kaier, Christian; Rieck, Katharina; Šimukovič, Eöema: Vignoli, Michela (2016): The Vienna Principles: A Vision for Scholarly Communication in the 21st Century. In: Mitteilungen der Vereinigung Österreichischer Bibliothekarinnen \& Bibliothekare,69 (3/4), 436-446.

42 Bauer, Bruno; Ferus, Andreas; Schilhan, Lisa (2016): Checkliste „Open Access Policies“: Analyse von Open Access Policies öffentlicher Universitäten in Österreich. . In: Mitteilungen der Vereinigung Österreichischer Bibliothekarinnen \& Bibliothekare, 69 (3/4), 447-475.
} 
Publikation in den betreffenden Zeitschriften für die Angehörigen der 34 im Konsortium vertretenen Einrichtungen kommt dem Lizenzmodell Springer Compact eine besondere Rolle zu. Während der Vertragslaufzeit von 2016 bis 2018 haben alle Forschenden und Studierenden der beteiligten Einrichtungen die Möglichkeit, mehr als 2.000 Zeitschriften zu nutzen und in über 1.600 HybridZeitschriften Open Access zu publizieren.

2016 wurden insgesamt 1.013 Fachbeiträge in Springer-Zeitschriften von Forschenden an österreichischen Einrichtungen nach dem Open Access-Modell veröffentlicht, die 27 der in der Kooperation vertretenen Einrichtungen zuzuordnen sind. ${ }^{43}$ Die Erweiterung der zur Verfügung stehenden Inhalte führte im vergangenen Jahr auch zu einer signifikanten Steigerung der Zugriffe auf Artikel der Springer-Zeitschriften.

\subsection{Bewilligung des Hochschulraumstrukturmittelprojektes Austrian Transition to Open Access}

Im Hinblick auf die vom Bundesministerium für Wissenschaft, Forschung und Wirtschaft (BMWFW) für 2016 angekündigte Neuausschreibung von Hochschulraumstrukturmittelprojekten wurde bereits ab Dezember 2015 von einer vom Forum Universitätsbibliotheken Österreichs (ubifo) eingesetzten Arbeitsgruppe ein Konzept für ein Projekt zu Open Access vorbereitet.

Die Ausschreibung für die Vergabe der Hochschulraumstrukturmittel durch das BMWFW für den Bereich der Verwaltungsinnovation erfolgte im Juli 2016. Dem Themenfeld Open Innovation/Digital Roadmap/Open Access/Open Research Data wurde dabei besondere Beachtung geschenkt:

„Eingereichte Projektanträge zu Open Access sollen sich an den Empfehlungen des Open Access Network Austria (OANA) orientieren, nach denen die gesamte wissenschaftliche Publikationstätigkeit in Österreich bis 2025 auf Open Access umgestellt werden soll. “44 Im Dezember 2016 wurde die Entscheidung des BMWFW über die Anträge im Bereich der Verwaltungsinnovation bekannt gegeben. Zu den förderwürdigen Projekten gehörte auch Austrian Transition to Open Access (AT2OA). Für den Förderzeitraum 2017 bis 2020 werden vom BMWFW finanzielle Mittel für die Umsetzung folgender Ziele bereitgestellt werden: (1) Analyse der Auswirkung einer Umstellung auf Open Access, (2) Finanzierung von Open Access Übergangsmodellen (Verlagsverträge), (3) Einrichtung von OA-Publikationsfonds und (4) Förderung von OA-Publikationen und alternativen OAPublikationsmodellen von Universitäten.

\footnotetext{
${ }^{43}$ OpenAPC (2016): Treemaps / Offsetting. View: Institution, Year: 2016, Country:_AUT. Online: https://treemaps.intactproject.org/apcdata/offsetting/\#institution/period=2016

${ }^{44}$ Bundesministerium für Wissenschaft, Forschung und Wirtschaft (2016). Hochschulraumstrukturmittel 2016 - Verwaltung: Ausschreibungstext. URL: http://unicontrolling.bmwfw.gv.at/index.php?option=com_content\&view=article\&id=53\&ltemid=175.
} 


\section{$5 \quad$ NS-Provenienzforschung}

Die Website der AG NS-Provenienzforschung der Vereinigung Österreichischer Bibliothekarinnen und Bibliothekare (VÖB) listet 14 wissenschaftliche Bibliotheken - die Österreichische Nationalbibliothek, die Wienbibliothek im Rathaus sowie die Universitätsbibliotheken von zehn öffentlichen Universitäten - auf, die NS-Provenienzforschung betreiben.

Am 20. Jänner 2016 fand an der Universität Klagenfurt die Restitution von Büchern statt, die in der nationalsozialistischen Ära kirchlichen Institutionen geraubt worden sind. Im Zuge der Überprüfung von 6.390 Bänden, die zwischen 1938 und 1953 an die ehemalige Studienbibliothek gelangt sind, konnten aufgrund von Hinweisen in Form von Stempel, Namenseinträgen und Widmungen 18 Werke bzw. 22 Bände als NS-Raubgut identifiziert werden.

„Restituimus, et nos vobis excusamus. - Wir haben nun restituiert und bitten Sie um Entschuldigung.“ - mit diesen Worten überreichte Rektor Oliver Vitouch die betreffenden Bände an die Vertreter der Benediktiner in St. Paul und der Barmherzigen Brüdern in St. Veit. Die Rechtsnachfolger der Pfarre St. Margarethen ob Töllerberg, der Jesuiten in St. Andrä und der Ursulinen in Klagenfurt verzichteten auf eine reale Restitution. ${ }^{45}$

2016 erfolgte auch an der Veterinärmedizinischen Universität Wien, wo von der Universitätsbibliothek seit 2011 NS-Provenienzforschung betrieben wird, die erste Restitution. 19 während der NS-Zeit unrechtmäßig erworbene veterinärmedizinische Bücher wurden am 18. Oktober 2016 in einem Festakt von Rektorin Petra Winter und Vizerektor Otto Doblhoff-Dier an die rechtmäßigen Erbinnen der Firma Kuppitsch restituiert. Diese übergaben die betretreffenden Bände im Anschluss als Geschenk an die Veterinärmedizinische Universität. ${ }^{46}$

\section{Einzelaktivitäten von Bibliotheken}

\subsection{Umbau der Universitätsbibliothek Graz}

Nachdem im Herbst 2015 nach Durchführung eines Architektenwettbewerbs das Siegerprojekt von Thomas Pucher ermittelt werden konnte, wurde 2016 mit der 28 Millionen Euro teuren Sanierung und Erweiterung der Universitätsbibliothek Graz begonnen. Im Zuge des Bauprojektes sind folgende Maßnahmen geplant: Abriss des Zubaus aus dem Jahr 1970, Bau eines unterirdischen Hörsaals mit 430 Sitzplätzen sowie Aufbau eines zweigeschossigen Glasquaders mit 200

\footnotetext{
${ }^{45}$ Kleine Zeitung (2016): Klagenfurt: NS-Restitution: Alpen-Adria-Uni gibt geraubte Bücher zurück. Die Universität Klagenfurt machte in ihren Bibliotheksbeständen 18 geraubte Bücher ausfindig, darunter eine 350 Jahre alte Ausgabe der "Technica Curiosa" von Kaspar Schott. In: Kleine Zeitung, 12.01.2016. Online:

http://www.kleinezeitung.at/kultur/4902762/Klagenfurt_AlpenAdriaUni-gibt-geraubte-Buecher-zurueck

${ }^{46}$ Dandler, Florian; Hausberger, Claudia (2016): Restitution geraubter Bücher an der Vetmeduni Vienna. In: Mitteilungen der Vereinigung Österreichischer Bibliothekarinnen \& Bibliothekare, 69 (3/4), 476-481
} 
Studierendenarbeitsplätzen, die zu den bestehenden 450 Plätzen des darunter liegenden historischen Lesesaals hinzukommen. ${ }^{47484950}$

Nach der Schließung der alten Bibliothek am 19. August 2016 und der Auslagerung der Bibliotheksbestände in Ausweichquartiere in Graz erfolgte am 19. September 2016 die Eröffnung des neuen Standorts der Universitätsbibliothek Graz in der Beethovenstraße 8, wo die neue Anlaufstelle für Benutzerinnen und Benutzer der Universitätsbibliothek Graz, an der Ortsleihe, Fernleihe, Lehrbuchsammlung, Informationsdienste, Publikationsservices und Arbeitsplätze für Benutzerinnen und Benutzer untergebracht sind. ${ }^{51}$

Im Herbst 2019 soll die Universitätsbibliothek Graz am alten Standort in neuem Ambiente ihren Betrieb wieder aufnehmen.

\subsection{Repositorien von Landesbibliotheken}

In den letzten Jahren haben sich die Repositorien an drei österreichischen Landesbibliotheken sehr gut entwickelt.

Die Zahl der digitalisierten und online zugänglichen Werke hat sich seit dem Start der Digitalen Landesbibliothek Österreich (DLOÖ) im Jahr 2010 von 25 auf 5000 im Jahr 2016 erhöht. Entsprechend gestiegen sind auch die Zugriffszahlen auf die digitalen Bestände der Oberderennsia. ${ }^{52}$ Mittlerweile wurde auch ein Crowdsourcing-Modul mit der Zielsetzung entwickelt, interessierten Benutzerinnen und Benutzer die Möglichkeit zu geben, Annotationen zu den Digitalisaten anzubringen. ${ }^{53}$

Die Wien Bibliothek im Rathaus startete 2011 mit der Bereitstellung der digitalisierten Adressbücher des Lehmann erfolgreich ihre digitale Wienbibliothek. Diese wird mittlerweile in folgenden drei Themenfeldern weiterentwickelt: Digitalisierung von Materialien zur Forschung über Wien, Digitalisierung von (Musik-)Handschriften sowie anlassbezogene Digitalisierung im Zusammenhang

\footnotetext{
${ }^{47}$ Swoboda, Norbert (2016): Spektakulärer Ausbau der Uni-Bibliothek in Graz. In: Kleine Zeitung, 28.10.2015. Online: http://www.kleinezeitung.at/steiermark/graz/4853735/Spektakulaerer-Ausbau-der-UniBibliothek-in-Graz

${ }^{48}$ Swoboda, Norbert (2016): Die Uni Graz rüstet sich für einen Großumbau. In wenigen Wochen beginnt der Umbau der Universitätsbibliothek in Graz. In: Kleine Zeitung, 03.04.2016. Online: http://www.kleinezeitung.at/steiermark/graz/4958911/KarlFranzensUniversitaet_Die-Uni-Graz-ruestet-sich-fuer-einen

${ }^{49}$ Kleine Zeitung (2016): Uni-Bibliothek Graz wird drei Jahre lang umgebaut. In wenigen Wochen startet der umfangreiche Umbau der Uni-Bibliothek Graz. 27,6 Millionen Euro werden investiert. In: Kleine Zeitung, 05.07.2016. Online: http://www.kleinezeitung.at/steiermark/bildung/5043844/Universitaet-Graz_UniBibliothek-Graz-wird-drei-Jahre-langumgebaut

${ }^{50}$ Schlacher, Werner (2016): Der Umbau der Universitätsbibliothek Graz - ein Bericht in drei Fortsetzungen. Teil 1: Wie alles begann. In: Mitteilungen der Vereinigung Österreichischer Bibliothekarinnen \& Bibliothekare, 69 (3/4), 422-435.

51 Universitätsbibliothek Graz (2016): Hereinspaziert! In: Universitätsbibliothek Graz: Neuigkeiten, 19.09.2016. Online: https://ub.uni-graz.at/de/neuigkeiten/detail/article/hereinspaziert-3/

52 Neuböck, Gregor (2016)r: Das Crowdsourcing-Modul der „Digitalen Landesbibliothek Oberösterreich“ (DLOÖ) . In: Bruno Bauer, Andreas Ferus und Josef Pauser (Hrsg.): Offen(siv)e Bibliotheken: Neue Zugänge, neue Strukturen, neue Chancen. 32. Österreichischer Bibliothekartag, Wien, 15.-18. September 2015. Wolfgang Neugebauer Verlag GesmbH: Graz-Feldkirch, 2016, S.283-288. (Schriften der Vereinigung Österreichischer Bibliothekarinnen und Bibliothekare 14)

53 Neuböck,. Gregor (2016): Kollaboration und Web 2.0. In: Bibliothek - Forschung und Praxis, 40 (3), 389-394.
} 
mit Jubiläen, Ausstellungen und Forschungskooperationen. 2014 wurde das Wien Geschichte Wiki, von der Wienbibliothek im Rathaus und dem Wiener Stadt- und Landesarchiv mit 32.000 Artikeln initiiert. Bereits im ersten Jahr wurden mehr als eine Million Zugriffe registriert. Strategisches Ziel ist es, die Inhalte der Digitalen Bibliothek und des Wiki stärker zusammenzuführen. ${ }^{54}$

Auch die Vorarlberger Landesbibliothek hat in jüngster Zeit mit volare ein Vorarlberger LAndesREpositorium gestartet. ${ }^{55}$ In Kooperation mit der Universität Wien erfolgte im Oktober 2015 die Präsentation der neuen Bilddatenbank mit 80.000 digitalisierten Fotos. ${ }^{56}$

\section{$7 \quad$ Ausbildung}

Das Curriculum für den interuniversitären Universitätslehrgang Library and Information Studies wurde 2013 aktualisiert.

2015/2016 wurde der Grundlehrgang, der jeweils an der Universität Wien, der Universität Graz und in Kooperation von der Universität Wien mit der Österreichischen Nationalbibliothek angeboten worden ist, von insgesamt 66 Studierenden erfolgreich abgeschlossen. 2016 wurde der Grundlehrgang 2016/2017 gestartet, der nur an der Universität Wien in Kooperation mit der Österreichischen Nationalbibliothek durchgeführt wird und an dem 23 Studierende teilnehmen.

\section{BID-Tagungen in Österreich}

\subsection{ODOK in Eisenstadt 2016}

Die ODOK fand von 27. bis 29. September 2016 statt und führte 186 Teilnehmerinnen und Teilnehmer nach Eisenstadt. Das Motto dieser traditionsreichen bibliothekarischen Fortbildungsveranstaltung, die gleichzeitig als 16. Österreichisches Online-Informationstreffen und als 17. Österreichischer Bibliothekartag durchgeführt worden ist, lautete „Dokument - Dienstleistung - Digital". Themenschwerpunkte der ODOK, die auf parallele Vorträge verzichtete, waren Open Access, Bibliothekssysteme und Bibliotheksservices, der Beitrag der Bibliotheken im Bereich von

\footnotetext{
${ }^{54}$ Eichinger, Anita; Pfoser, Alfred (2016): Wienbibliothek Digital - Strategie und Profilbildung. In: Bruno Bauer, Andreas Ferus und Josef Pauser (Hrsg.): Offen(siv)e Bibliotheken: Neue Zugänge, neue Strukturen, neue Chancen. 32. Österreichischer Bibliothekartag, Wien, 15.-18. September 2015. Wolfgang Neugebauer Verlag GesmbH: Graz/Feldkirch, 2016, S.41-48. (Schriften der Vereinigung Österreichischer Bibliothekarinnen und Bibliothekare 14)

${ }^{55}$ Sauter-Sternik, Günter (2016): VOLARE - VOrarlberger LAndesREpositorium. In: Bruno Bauer, Andreas Ferus und Josef Pauser (Hrsg.): Offen(siv)e Bibliotheken: Neue Zugänge, neue Strukturen, neue Chancen. 32. Österreichischer Bibliothekartag, Wien, 15.-18. September 2015. Wolfgang Neugebauer Verlag GesmbH: Graz/Feldkirch, 2016, S.171-176. (Schriften der Vereinigung Österreichischer Bibliothekarinnen und Bibliothekare 14)

${ }^{56}$ Gasser, Michael (2016): Eine digitale Zukunft für alte Fotos. Fast eine Million Fotos im Keller: Landesbibliothek macht Bildarchiv digital zugänglich. In: Vorarlberger Nachrichten, 03./04.10.2015. Online: https://vlb.vorarlberg.at/fileadmin/vlb/downloads/was_passiert/veranstaltungen/2015/pressevolare/20151003_VNA_20151003_005.pdf
} 
Publikationsberatung und Forschungsdatenmanagement, die digitale Welt der I\&D-Dienstleister sowie der digitale Wandel in Bibliotheken. ${ }^{57}$

\subsection{COAR in Wien 2016}

Am 12. und 13. April 2016 fanden die Jahrestagung und daran anschließend eine Konferenz von COAR (Confederation of Open Access Repositories) statt. 80 Teilnehmerinnen und Teilnehmern kamen an der Universität Wien zusammen, um sich dem Thema der internationalen Zusammenarbeit in technischer, inhaltlicher und organisatorischer Hinsicht zu widmen. Insbesondere Fragen zur Weiterentwicklung im Bereich Open Access und Langzeitarchivierung wurden erörtert. ${ }^{58}$

\subsection{Weitere BID-Veranstaltungen in Österreich}

Die 2010 initiierte Tagungsreihe Digitale Bibliothek fand am 25. und 26. Februar 2016 zum sechsten Mal statt. Zum Generalthema Vernetzte Welten -Digitales Kulturerbe als öffentliches Gut kamen mehr als 100 Teilnehmerinnen und Teilnehmer aus Österreich und den Nachbarländern nach Graz, um Fragen zu Konservierung, Pflege und Erschließung von digitalen Sammlungen zu erörtern. Der Schwerpunkt lag dabei auf Vernetzung, Kollaboration und kreativer Wiederverwendung von Daten. ${ }^{59}$

Am 20. Mai 2016 präsentierte Bundespräsident Heinz Fischer in den Räumen der österreichischen Präsidentschaftskanzlei biografiA: Lexikon österreichischer Frauen der Öffentlichkeit. Das von Ilse Korotin herausgegebene vierbändige Werk umfasst rund 6.500 Biografien österreichischer Frauen von der Römerzeit bis zur Gegenwart, einschließlich Geburtsjahr 1938. Aufgenommen sind Frauen, deren Wirken in Politik, Gesellschaft, Wissenschaft, Kultur und Geschichte bemerkenswert ist, wobei der geografische Rahmen des Werkes auf das heutige Österreich begrenzt wurde. ${ }^{60}$ Das Lexikon, das für alle interessierten Personen auch als Open Access-Publikation kostenfrei zugänglich ist ${ }^{61}$, verzeichnet auch an die 100 Frauen mit der Berufsbezeichnung Bibliothekarin.

Am 13. Juni 2016 fand an der Universitätsbibliothek der Medizinischen Universität Wien das 6. Treffen des Arbeitskreises österreichischer Medizinbibliothekarinnen und -bibliothekare statt. Der Arbeitskreis ist innerhalb der Arbeitsgemeinschaft für Medizinisches Bibliothekswesen (AGMB)

\footnotetext{
57 OGDI (2016): Das war die ODOK 2016. In: oegdi-news, 04.10.2016. Online: http://us13.campaignarchive2.com/?u=c6f5035a12ea393aaa66b8291\&id=0be3b321fe \&e=2f85cb18df

58 Semanek, Brigitte; Finta, Anna-Maria; Blumesberger, Susanne (2016): Jahrestagung und Konferenz der COAR (Confederation of Open Access Repositories) 2016 (Wien, 12.-13. April 2016) . In: Mitteilungen der Vereinigung Österreichischer Bibliothekarinnen \& Bibliothekare, 69 (2), 329-336.

59 Blumesberger, Susanne; Gründhammer, Veronika; Koch, Gerda; Pipp, Eveline; Plößnig, Veronika (2016): 6. Tagung „Digitale Bibliothek“ - (Graz, 26. Februar 2016) . In: Mitteilungen der Vereinigung Österreichischer Bibliothekarinnen \& Bibliothekare, 69 (2), 319-328.

60 Bauer, Bruno; Blumesberger, Susanne; Heindl, Waltraud; Korotin, Ilse; Stumpf-Fischer, Edith (2016): Präsentation eines vierbändigen Lexikons mit rund 6.500 Biografien österreichischer Frauen durch Bundespräsident Heinz Fischer in den Räumen der Präsidentschaftskanzlei in Wien am 20. Mai 2016. In: Mitteilungen der Vereinigung Österreichischer Bibliothekarinnen \& Bibliothekare, 69 (3/4), 281-303.

61 Korotin, Ilse (Hrsg., 2016): biografiA: Lexikon österreichischer Frauen. 4 Bände. Hrsg. von Ilse Korotin. Online: http://www.boehlau-verlag.com/978-3-205-79590-2.html
} 
angesiedelt, der als Verein zur Förderung des medizinischen Bibliotheks- und Informationswesens organisiert ist und der ca. 500 Mitglieder in Deutschland, Österreich und der Schweiz aufweist. ${ }^{62}$

Die Jahrestagung des Arbeitskreises kritischer Bibliothekarinnen und Bibliothekare (KRIBIBI), die am 4. und 5.November 2017 in Wien stattfand, war dem Thema Armut und Bibliotheken - eine Herausforderung gewidmet. ${ }^{63}$

In der Woche von 3. bis 9. Oktober 2016 fand die vom Büchereiverband Österreichs (BVÖ) initiierte und hauptverantwortlich durchgeführte Veranstaltungsreihe Österreich liest. Treffpunkt Bibliothek statt, in deren Rahmen von zahlreichen Bibliotheken in Österreich wiederum zu einer Fülle an Lesungen, Literaturwanderungen, Bilderbuchkinos, Lesenächten, Literatur-Cafés, Buchausstellungen und vielen weiteren Aktivitäten, die Lust aufs Lesen machen, eingeladen worden ist.

\section{$9 \quad$ Bibliothekspolitik}

\subsection{Publikationen der VÖB}

2016 erschienen drei Ausgaben der von der VÖB herausgegebenen Zeitschrift Mitteilungen der Vereinigung Österreichischer Bibliothekarinnen und Bibliothekare. Seit Ausgabe 2016/1 wird die Publikationsplattform Open Journal Systems (OJS) mit Unterstützung der Universitätsbibliothek Wien genutzt. ${ }^{64}$ Ausgabe 2016/2 war dem Schwerpunktthema Gender \& Diversity gewidmet. ${ }^{65}$

2016 erschien als Band 14 der Schriften der Vereinigung Österreichischer Bibliothekarinnen und Bibliothekare auch der Tagungsband zum 32. Österreichischen Bibliothekartag, der von 15. bis 18. September 2015 in Wien stattgefunden hat. ${ }^{66}$

\subsection{Pressearbeit der VÖB}

2016 wurden die Dienste von APA OTS, einem Dienstleister zur Verbreitung von multimedialen Presse- und Marketinginformationen an Medien, Meinungsbildner und Pressestellen im In- und Ausland, von der VÖB zur Information über die ODOK in Eisenstadt genutzt. ${ }^{67}$

\footnotetext{
62 Bauer, Bruno (2016): 6. Treffen des Arbeitskreises österreichischer Medizinbibliothekarinnen und -bibliothekare (Wien, 13. Juni 2016) . In: Mitteilungen der Vereinigung Österreichischer Bibliothekarinnen \& Bibliothekare, 69 (3/4), 346-350.

63 Hamann, Nikolaus (2016): Jahrestagung 2016 Kurzbericht. „Armut und Bibliotheken - eine Herausforderung“. Jahrestagung des Arbeitskreises kritischer Bibliothekarinnen und Bibliothekare (KRIBIBI) am 4. und 5. November 2016. In: KRIBIBI: Arbeitskreis kritischer Bibliothekarinnen und Bibliothekare, 12.11.2016. Online: http://www.kribibi.at/aktivitaeten/seminare/135-jahrestagung-2016-kurzbericht

${ }^{64}$ Redaktion der Mitteilungen der VÖB (2016): OJS ante portas! Editorial. In: Mitteilungen der Vereinigung Österreichischer Bibliothekarinnen \& Bibliothekare, 69 (1), 6-8.

${ }^{65}$ Bargmann, Monika (2016): Schwerpunktthema „Gender \& Diversity“. Editorial. In: Mitteilungen der Vereinigung Österreichischer Bibliothekarinnen \& Bibliothekare, 69 (2), 185-187.

${ }^{66}$ Bauer, Bruno; Ferus, Andreas; Pauser, Josef (Hrsg., 2016): Offen(siv)e Bibliotheken : neue Zugänge, neue Strukturen, neue Chancen : 32. Österreichischer Bibliothekartag Wien, 15.-18. September 2015. Graz/Feldkirch : Wolfgang Neugebauer Verlag GesmbH, 2016. (Schriften der Vereinigung Österreichischer Bibliothekarinnen und Bibliothekare 14)
} 


\subsection{Young Information Scientist}

Auf Initiative von Otto Oberhauser wurde 2016 mit Young Information Scientist ${ }^{68}$ eine neue Open Access-Zeitschrift für die Fachgebiete Informationswissenschaft und Bibliothekswesen gegründet, in der im ersten Erscheinungsjahr fünf Beiträge veröffentlicht worden sind. ${ }^{69}$ Die Zeitschrift wird getragen vom Verein zur Förderung der Informationswissenschaft (VFI), der von 2006 bis 2015 jährlich den VFI-Förderpreis für hervorragende wissenschaftliche Abschlussarbeiten im Bereich der Informationswissenschaften herausgegeben hat. ${ }^{70}$

\subsection{BAM Austria}

Bibliotheken, Archive, Museen Österreichs (BAM) wurde 2003 als Round Table gegründet, an dem sich regelmäßig Vertreterinnen und Vertreter der Dachorganisationen der Bibliotheken, Dokumentationsstellen, Archive, AV-Archive, Museen und zusätzlich Vertreterinnen und Vertreter einzelner Institutionen beteiligen, um sich unabhängig vom Wissensgebiet gegenseitig über neue Entwicklungen zu informieren, Erfahrungen auszutauschen und in der Öffentlichkeit für die gemeinsame Sache einzutreten. ${ }^{71}$ Für 2016 wurde aufbauend auf eine deutsche Initiative, die das Ziel verfolgt, die Gemeinsame Normdatei (GND) auch für Archive und Museen zu öffnen, die GND zum Leitthema von BAM Austria 2016 erhoben. Um fundierte Erkenntnisse über die Praxis der Objekterschließung an diesen Einrichtungen zu erhalten, wurde eine Projektarbeit im Rahmen des Universitätslehrgangs Library and Information Studies an der Österreichischen Nationalbibliothek angeregt. Von 198 kontaktierten österreichischen Institutionen des BAM-Bereichs antworteten 62 (31\% Rücklaufquote), davon 20 Bibliotheken, 14 Archive, 7 Museen und 21 kombinierte Einrichtungen. Von den an der Befragung beteiligten Institutionen verfügen 75\% über ein kontrolliertes (normiertes) Vokabular, allerdings verwenden nur 35\% die GND. Besonders wertvoll

\footnotetext{
${ }^{67}$ APA OTS (2016): VÖB lädt zur Tagung "ODOK 2016" nach Eisenstadt. Bibliotheken gestalten die E-Medien-Zukunft. In: APA OTS, OTS0189, 22.09.2016. Online: https://www.ots.at/presseaussendung/OTS_20160922_OTS0189/voeb-laedt-zurtagung-odok-2016-nach-eisenstadt-bibliotheken-gestalten-die-e-medien-zukunft

68 Young Information Scientist (2016): YIS. Wien : Verein zur Förderung für Informationswissenschaft (VFI), 2016-. Online: https://yis.univie.ac.at/

69 Oberhauser, Otto (2016): Young Information Scientist - eine neue Open Access-Zeitschrift für die Fachgebiete Informationswissenschaft und Bibliothekswesen. In: Mitteilungen der Vereinigung Österreichischer Bibliothekarinnen \& Bibliothekare, 69 (3/4), 482-486.

70 Oberhauer, Otto (2016): Vergabe des VFI-Förderungspreises 2015. In: Mitteilungen der Vereinigung Österreichischer Bibliothekarinnen \& Bibliothekare, 69 (3/4), 109.

${ }^{71}$ Weigel, Harald (2016): BAM Austria - Einheit in Vielfalt? Kulturbewahrer und Informationsvermittler: einzeln stark gemeinsam stärker. In: Bruno Bauer, Andreas Ferus und Josef Pauser (Hrsg.): Offen(siv)e Bibliotheken: Neue Zugänge, neue Strukturen, neue Chancen. 32. Österreichischer Bibliothekartag, Wien, 15.-18. September 2015. Wolfgang Neugebauer Verlag GesmbH: Graz/Feldkirch, 2016, S.67-82. (Schriften der Vereinigung Österreichischer Bibliothekarinnen und Bibliothekare 14)
} 
war auch das Feedback zur Themenkomplex Open Data. ${ }^{72}$ Dieses Thema steht auch auf der Agenda von BAM für 2017.

\subsection{Memory of Austria}

Seit 2014 wird vom österreichischen Memory of the World-Nationalkomitee eine Liste besonders relevanter und typischer Objekte des österreichischen Dokumentenerbes erstellt, in der Dokumente und Sammlungen aus Bibliotheken, Archiven, Museen und audiovisuellen Archiven verzeichnet sind. ${ }^{73}$ Am 17. November 2016 erfolgten 22 Neuaufnahmen in das Österreichische Nationale Memory of the World Register, das mittlerweile insgesamt 41 österreichische Einträge aufweist. Die Neuaufnahmen sind folgenden Institutionen zuzuordnen: Österreichische Nationalbibliothek (United States Information Service (USIS) - Fotoarchiv; Briefe von Hannah Arendt an Günther Anders (19391970); Erich Lessing: Internationale Reportagefotografie 1945-1970; Erst- und Frühdrucksammlung Anthony van Hoboken; Joseph Daniel von Huber - Vogelschauansicht von Prag (1769); Nachlass Ingeborg Bachmann; Sammlung von Flugblättern und Flugschriften zur Revolution 1848 in der Habsburgermonarchie; Wolfgang Lazius: Typi chorographici), Österreichisches Staatsarchiv (Abschied des Reichstages von Augsburg 1555 - Augsburger Religionsfriede; Archiv der Wiener Zeitung; Bannandrohungsbulle Martin Luther „Exsurge Domine“; Josephinische Landesaufnahme der Habsburgermonarchie; Martin Luthers 95 Thesen; Stiftungshofbuchhaltung; Westfälische Friedensverträge), Wienbibliothek im Rathaus (Karl Kraus-Archiv), Österreichische Zentralbibliothek für Physik (Nachlass Hans Thirring), Österreichische Nationalbibliothek und Österreichisches Staatsarchiv (Die mittelalterliche Handschriftensammlung des ehemaligen Benediktinerstiftes Mondsee in Oberösterreich), Technisches Museum Wien mit Österreichischer Mediathek und Österreichischer Rundfunk (Historische Radioaufnahmen RAVAG und Rot-Weiß-Rot; Nachlass Josef Ressel; Tonaufnahmen der Österreichischen Mediathek 1965-2000) sowie Wienbibliothek im Rathaus und Österreichische Mediathek (United States Information Service (USIS) - Radiosendungen). ${ }^{74}$

\footnotetext{
72 Marković, Barbara; Kmyta, Olga; Sucker, Irina (2016): Objekterschließung an Bibliotheken, Museen und Archiven in Österreich. Ergebnisse einer Erhebung. In: Mitteilungen der Vereinigung Österreichischer Bibliothekarinnen \& Bibliothekare, $69(3 / 4), 414-421$.

${ }^{73}$ Schüller, Dietrich; Hubert, Rainer (2016): Memory of the World: Das UNESCO Programm zur Sicherung des Dokumentenerbes. In: Bruno Bauer, Andreas Ferus und Josef Pauser (Hrsg.): Offen(siv)e Bibliotheken: Neue Zugänge, neue Strukturen, neue Chancen. 32. Österreichischer Bibliothekartag, Wien, 15.-18. September 2015. Wolfgang Neugebauer Verlag GesmbH: Graz/Feldkirch, 2016, S.59-65. (Schriften der Vereinigung Österreichischer Bibliothekarinnen und Bibliothekare 14)

74 Österreichische UNESCO-Kommission (2016): Memory of Austria - Verleihung der Urkunden an die Neuaufnahmen 2016. Online: https://www.unesco.at/kommunikation/dokumentenerbe/artikel/article/memory-of-austria-verleihung-derurkunden-an-die-neuaufnahmen-2016/
} 


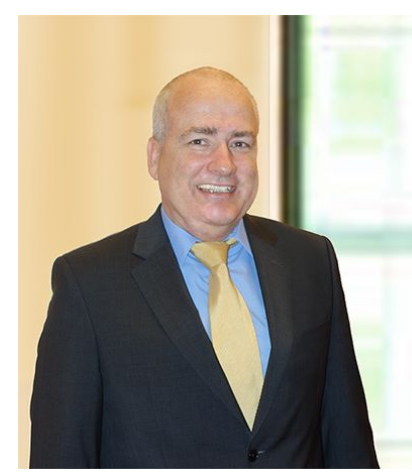

Mag. Bruno Bauer

Leiter der Universitätsbibliothek der Medizinischen Universität Wien

Währinger Gürtel 18-20

A-1097 Wien

Österreich

bruno.bauer@meduniwien.ac.at

orcid.org/0000-0002-4729-331X 